\title{
Psychiatric disorders in HIV-positive individuals in urban Uganda
}

\section{AIMS AND METHOD}

The study examined the prevalence of psychiatric disorders in people with HIV/AIDS attending the AIDS Support Organisation (TASO) clinic at Mulago Hospital, Kampala, Uganda and the preparedness of AIDS counsellors to deal with mental disorders. Forty-six patients were interviewed using the Mini International Psychiatric Interview to ascertain DSM-IV diagnoses. All 15 counsellors working at the clinic were interviewed.

\section{RESULTS}

The total prevalence of psychiatric disorder was 82.6 (38 out of 46 patients). Depressive and anxiety disorders were common. Nonaffective psychoses were present in eight patients $(17.4 \%)$, bipolar affective disorder in eight (17.4\%) and major depression with melancholic features in five $(10.9 \%) ; 8$ (13\%) had current suicidal thoughts. None of the people with psychiatric disorders were receiving mental health treatment. The prevalence of disorder as estimated by the counsellors ranged from 0 to $33 \%$. Only one counsellor had received any formal training in mental disorders and only two thought that they could deal with these if they arose. The attitudes of counsellors towards people with mental disorders were mixed, but most believed that they should be trained to provide care.
CLINICAL IMPLICATIONS
There is a need to provide additional mental health services to theTASO clinic through appropriate training of TASO counsellors to improve their awareness of psychiatric disorders, delivery of some psychological therapies and liaison with the psy- chiatric services at Mulago Hospital, in addition to public mental health education. The psychiatric disorders experienced by those attending the clinic might put them at greater risk of contracting HIV/AIDS.

There is a well-documented strong association between depressive disorders and HIV/AIDS (e.g. Maj et al, 1994; Perkins et al, 1994; Judd et al, 1997). High levels of depression are seen in people with HIV/AIDS attending primary care clinics (Savetsky et al, 2001) and in HIV medical clinics (Lyketsos et al, 1994a). There appears to be a sustained rise in depressive symptoms as AIDS develops (Lyketsos et al, 1994b). Depression is also associated with HIV-related risk behaviours among those without HIV (Kelly et al, 1993). Nevertheless, most persons affected by HIV/AIDS in Uganda do not receive any care for their mental health problems.

The early cases of AIDS were described in Uganda, but there has been little work examining the existence of psychiatric disorders in AIDS/HIV in that country. The national rate of HIV at the end of 2003 was $4.1 \%$ (UNAIDS, 2004), but is as high as $13 \%$ in war-torn northern Uganda. Of adult deaths at Mulago Hospital, Kampala, $60-70 \%$ are attributable to HIV. Wilk \& Bolton (2002) investigated how people in two districts of Uganda (Masaka and Rakai), which have been severely affected by HIV, perceive the mental health affects of the disease. The individuals interviewed described two independent depression-like syndromes resulting from the HIV epidemic and rates of depressive disorder were estimated to be $21 \%$ in these districts (Bolton et al, 2004). Anecdotal evidence also links HIV/AIDS to suicide in Uganda (Musisi et al, 2001; Kinyanda \& Musisi, 2002).

A previous study carried out in the AIDS Support Organisation (TASO) clinic at Mulago Hospital, Kampala using the General Health Questionnaire (GHQ-28; Goldberg, 1978) found estimated levels of psychiatric disorder of 74\% (Kinyanda, 1998). No interview measures were used in this study and it is possible that the levels of psychiatric morbidity were overestimated owing to the tendency of the GHQ to detect false-positives with high numbers of physical symptoms (Goldberg, 1978). The present study was designed to examine further the rates of psychiatric disorders in this clinic and to examine the preparedness of the clinic HIV counsellors to deal with psychiatric problems.

\section{The AIDS Support Organisation (TASO)}

The study was carried out in the AIDS Support Organisation (TASO) based in Mulago Hospital, Kampala, Uganda. The AIDS Support Organisation was the first HIV/AIDS non-governmental organisation to be created in Uganda in 1987 and has since grown into the largest such HIVrelated organisation with over 12 centres all over Uganda, and many more monthly outreach clinics in most districts. The organisation offers the following services to its patients:

(a) HIV testing (not carried out atTASO but at a local testing facility);

(b) Counselling as often as requested to allow people to lead normal lives and live positively;

(c) Medical treatment for patients and their children for opportunistic infections, etc.;

(d) Food in the form of cornflour and vegetable oil (supplied by the US Agency for International Development);

(e) Tea while at clinics; 
(f) Antiretrovirals;

(g) Occasional referrals to psychiatric services.

original papers
As well as the above services, a group of HIV-positive musicians and dancers entertain communities and teach them about safe sex and the dangers of HIV, as well as encouraging people to embrace and respect those who are already infected.

The AIDS Support Organisation has been established as a public service, available to anyone free of charge. Therefore, there is large variation between the social and financial background of the patients attending clinics.

The aims in the study were to:

(a) estimate the prevalence of mental illness in those attending theTASO clinic in Mulago, and to obtain specific diagnoses for these patients;

(b) compare the counsellors'estimation of the prevalence of mental illness with that found using the standardised psychiatric interview;

(c) examine the attitudes and beliefs of theTASO counsellors regarding the issue of mental illness.

\section{Method}

There were two components to the study: the patient survey and counsellor interviews.

\section{Patient survey}

Every fifth patient attending the TASO clinic was invited to participate in the study. Consent was obtained and each patient signed or thumb printed a consent form. Basic demographic details were recorded and the patients were interviewed using the Mini International Neuropsychiatric Interview (MINl; Sheehan et al, 1998), a standardised clinical diagnostic interview schedule for DSM-IV (American Psychiatric Association, 1994). Diagnoses are made according to diagnostic algorithms, which require a fixed number of symptoms, a minimum duration of symptoms causing distress and a definite impairment of social functioning as a result of symptoms. Translated versions of the MINI have been used in previous community-based studies in Uganda (Ovuga et al, 2005). The interviews were carried out in English or Luganda, the local language, under the supervision of E.O., H.P. and two Ugandan medical students. Interviews were carried out in private rooms in the clinic.

Using the Kish (1965) population sampling formula, a sample size of 48 was decided upon. This was based on a $20 \%$ estimated prevalence of mental illness at a population size of 300 patients over a 3 week period and a confidence interval of 0.99 .

\section{Counsellor interviews}

All 15 counsellors ( 3 males, 12 females) based at the Mulago TASO clinic agreed to take part in the study. The interviews were carried out by H.P. and conducted in English (all counsellors were fluent in English). The interview was specially designed for the study and consisted of the following questions:

(a) What issues regarding mental health do you think you encounter most in HIV-positive patients?

(b) Of the six patients you see every clinic day, how many of them do you think have a mental illness?

(c) What would you do if you thought someone was mentally ill?

(d) What aspects (if any) of mental illness are you uncomfortable dealing with?

(e) Were you trained to deal with mental illness?

(f) Do you think counselling is beneficial for patients with mental illness?

(g) Do you think HIV-positive patients should receive additional psychiatric counselling?

All responses were recorded and the content analysed.

\section{Results}

\section{Patient survey}

All 46 patients who were approached agreed to participate; $24(52.2 \%)$ were female and the mean age of the sample was 36.6 years (range $22-56$ years). Thirty-one (67.4\%) were unemployed; the remainder had some form of work, ranging from peasant farmer $(n=2)$ to roadside or market vendor $(n=3)$ to business people and local politicians $(n=2)$. All were HIV-positive and had been known to be so for a mean of 4 years (range $0-12$ years); 34 (74\%) had been known to be HIV-positive for less than 5 years. Eighteen (39\%) were known to have an AIDS-defining illness. All but 1 had active physical symptoms and $11(24 \%)$ had tuberculosis. None of the sample was on psychotropic medication or in contact with psychiatric services.

Thirty-eight (82.6\%) of the sample had a psychiatric disorder according to DSM-IV diagnoses (Table 1). One had an adjustment disorder only and one a diagnosis of

Table 1. Prevalence of DSM-IV diagnoses in those attending the AIDS Support Organisation (TASO) clinic $(n=46)$

\begin{tabular}{lc} 
DSM-IV category & $n(\%)$ \\
\hline Adjustment disorder & $1(2.2)$ \\
Dysthymia & $4(8.7)$ \\
Major depression & $25(54.3)$ \\
Bipolar disorder & $8(17.4)$ \\
Panic & $15(32.6)$ \\
Agoraphobia & $11(23.9)$ \\
Social phobia & $5(10.9)$ \\
OCD & $2(4.3)$ \\
PTSD & $9(19.6)$ \\
Alcohol misuse & $2(4.3)$ \\
Substance misuse & $0(0.0)$ \\
Non-affective psychosis & $8(17.4)$ \\
& \\
No diagnosis & $8(17.4)$ \\
\hline
\end{tabular}

OCD, obsessive-compulsive disorder; PTSD, post-traumatic stress disorder. 
dysthymia only. The most common diagnostic categories were major depression (54.3\%) and panic disorder (32.6\%). Multiple diagnoses were common (23 patients had multiple diagnoses $-60.5 \%$ of those with any diagnosis).

Seven people had a diagnosis of major depression only, in seven there was comorbidity with anxiety disorder, seven with non-affective psychosis and four cases were the depressive component of a bipolar affective disorder. Three had panic disorder only and two had mania in the context of a bipolar disorder only.

The disorders were generally severe. Eight (17.4\%) had a non-affective psychosis, eight (17.4\%) had a bipolar affective disorder and five (10.9\%) had major depression with melancholic features. Six (13.0\%) had current suicidal thoughts.

\section{Counsellor interviews}

The prevalence of psychiatric problems as estimated by the counsellors was generally small. Seven (46.7\%) believed they saw no people with psychiatric problems in the course of their clinics, five thought they saw one person with a psychiatric problem in every six they counselled and three thought they saw two people in every six. Five counsellors were aware that they saw people with psychoses; four thought they saw people with depression and six thought they saw people with anxiety. Some counsellors used other terms, such as memory loss $(n=4)$, stress $(n=2)$, violence $(n=2)$, impatience $(n=2)$, restlessness $(n=1)$, being silent or isolated $(n=2)$.

Many were uncomfortable with the idea of seeing people with psychiatric problems, usually because of the fear of violence $(n=8)$ or unpredictability $(n=1)$. Three counsellors said they felt comfortable with all the clients they saw, but two felt uncomfortable with 'anything medical' and one was adamant that seeing people with psychiatric disorders was not a job for counsellors. Only one said that they had been trained to deal with people with mental disorders, the remainder said that they had no specific training, but four thought they had learned during their jobs. Only two thought that they could deal specifically with psychiatric problems when they arose in their clients, the remainder wished to make a referral to a psychiatrist $(n=6)$ or put an entry in the notes $(n=7)$.

Most of the counsellors $(n=10)$ believed that counselling could be beneficial for some people with psychiatric disorders but not for all. One believed that counselling the relatives was important, but not the clients. Three believed that drugs were the best means of treating psychiatric problems. Four counsellors did not think that HIV-positive patients should receive extra counselling, but the remainder believed that it might be useful, often qualifying this by statements that it should apply to those in need or that counsellors should be trained to deal with people with psychiatric disorders.

\section{Discussion}

The survey suggests that the high prevalence of psychiatric disorders found by Kinyanda (1998) in people attending the TASO clinic in Mulago Hospital, Kampala was not an overestimate. It confirms that the level of psychiatric disorders is high and that many of these disorders are severe. Depressive and anxiety symptoms are common and often comorbid with psychoses. Current suicidal ideas were found in $13 \%$. It is possible that the MINI may overestimate the prevalence of disorders, but H.P. was present at all interviews and observed clear evidence of disorder in many, especially those with severe disorders. Even if the estimates for non-psychotic disorder were overestimated by $50 \%$, the rates of disorder would remain high. None of these disorders was considered to be the direct neuropsychiatric consequences of HIV/AIDS, and it is possible that some of the disorders might have contributed to the high-risk behaviour that may have resulted in developing HIV positivity.

This high level of psychiatric disorders (82.6\%) does not correspond with the prevalence of disorder as estimated by the counsellors, which ranged from 0 to $33 \%$. They might have been aware of some of the patients with more florid psychoses, but most of the disorders were not recognised.

The need for psychiatric treatment is high among those attending the clinic, but none was receiving any form of psychiatric treatment or care at the time of the survey. However, the counsellors were ill-equipped to deal with the level of disorder seen, as only one had received any formal training with people with mental disorders and only two thought that they would deal with such disorders if they arose. The attitudes of counsellors towards people with mental disorders are mixed, but most believed that they should be trained to provide care.

The observed discrepancy between need for psychiatric services among patients, the level of recognition among counsellors and the provision of mental healthcare to patients might stem from the concept of mental illness in Uganda. Mental illness is widely seen as synonymous with psychosis and believed to be caused by witchcraft, supernatural forces and the actions of evil spirits. The provision of mental healthcare to people with HIV/AIDS will require public mental health education, promotion of psychiatry in health sciences education, collaboration with traditional healers and addressing the ethical concerns and possible stigmatisation of persons with mental illness.

This study reveals the need to provide additional mental health services to the TASO clinic. This could be achieved by provision of additional training of the willing TASO counsellors to assist in the detection of people with psychiatric disorders, the provision of some psychological therapies and liaison with the psychiatric services already provided at Mulago Hospital. The services provided by TASO are highly regarded, but could be improved by recognition of the high level of psychiatric morbidity among those attending the clinic and the need to provide increased care and liaison for psychiatric disorders. original papers 


\section{Declaration of interest}

original

papers

\section{References}

AMERICAN PSYCHIATRIC

ASSOCIATION (1994) Diagnostic and

Statistical Manual of Mental Disorders (4th edn) (DSM-IV). Washington, DC: APA.

BOLTON, P.,WILK, C. M. \& NDOGONI, L. (2004) Assessment of depression prevalence in rural Uganda using symptom and function criteria. Social Psychiatry and Psychiatric Epidemiology, 39, 442-447.

GOLDBERG, D. P. (1978) Manual of the General Health Questionnaire.Windsor, UK: NFER-Nelson.

JUDD, F., MIJCH, A., McCAUSLAND, J., et al (1997) Depressive symptoms in patients with HIV infection: a further exploration. Australia and New Zealand Journal of Psychiatry, 31, 862-868.

KELLY, J. A., MURPHY, D. A., BAHR, G. R., et al (1993) Factors associated with severity of depression and highrisk sexual behavior among persons diagnosed with human immunodeficiency virus (HIV) infection. Health Psychology, 12, 215-219.
KINAYADA, E. (1998) Frequency with which psychiatric disorder is associated with a positive HIV-I serostatus as seen in persons attending aTASO clinic in Mulago. South African Medical Journal, 88, 1178.

KINYANDA, E. \& MUSISI, S. (2002) The psychological and social problems of HIV-sero-positive adolescents and their parents/guardians as seen at Mildmay Center, Uganda. African Psycare Research Organization Newsletter, June.

KISH, L. (1965) Survey Sampling. New York: Wiley.

LYKETSOS, C. G., HANSON, A. L. FISHMAN, M., et al (1994a) Screening for psychiatric disorders in an HIV medical clinic: the importance of a psychiatric presence. International Journal of Psychiatry in Medicine, 24, 103-113.

LYKETSOS, C. G., HOOVER, D. R., GUCCIONE, M., etal(1994b) Changes in Depressive Symptoms as AIDS develops. American Journal of Psychiatry, 153, 1430-1437.
MAJ, M., JANSSEN, R., STARACE, F., et al (1994) WHO neuropsychiatric AIDS study, cross-sectional phase l: study design and psychiatric findings.

Archives of General Psychiatry, $\mathbf{5 1}$ $39-49$

MUSISI, S., KINYANDA, E. \& NAKASUJJA, N. (2001) The nature, pattern and correlates of psychological behavioural risk factors in HIV transmission in Uganda. African Psycare Research Organization Newsletter, November.

OVUGA, E. BOARDMAN, J. \& WASSERMAN, D. (2005) The prevalence of depression in two districts of Uganda. Social Psychiatry and Psychiatric Epidemiology, 40, 439-445.

PERKINS, D. O., STERN, R. A., GOLDEN,

R. N., et al (1994) Mood disorders in HIV infection: prevalence and risk factors in a nonepicenter of the AIDS epidemic. American Journal of Psychiatry, 151 233-236

SAVETSKY, J. B., SULLIVAN, L. M. CLARKE, J., et al (2001) Evolution of depressive symptoms in human immunodeficiency virus-infected patients entering primary care. Journa of Nervous and Mental Disease, 189, $76-83$

SHEEHAN, D.V., LECRUBIER,Y. HARNETT-SHEEHAN, K., et al (1998) The Mini International Neuropsychiatric interview. The development and validation of a structured diagnostic psychiatric interview. Journal of Clinical Psychiatry, 59 (suppl. 20), 20-23.

UNAIDS (2004) Uganda: Epidemiological Fact Sheets on HIVI AIDS and SexuallyTransmitted Infections. 2004 Update. http:// www.unaids.org

WILK, C. M. \& BOLTON, P. (2002) Loca perceptions of the mental health effects of the Uganda acquired immunodeficiency syndrome epidemic. Journal of Nervous and Mental Disease, 190, 394-397.

Harry Petrushkin Medical Student, King's College London School of Medicine, *Jed Boardman Senior Lecturer in Social Psychiatry, Health Services Research Department, Institute of Psychiatry, King's College London, De Crespigny Park, London SE5 8AF, UK, e-mail: jed.boardman@scmh.org.uk, Emilio Ovuga Professor of Psychiatry, Makerere University, Kampala, Uganda 